\title{
Exploring potential synergistic effects between dietary adenosine and inosine monophosphate on growth performance and acute stress-induced immune responses of hybrid striped bass Morone chrysops x Morone saxatilis
}

\begin{abstract}
Two experiments were carried out to evaluate the effects of dietary adenosine monophosphate (AMP) and inosine monophosphate (IMP) on growth performance and acute stress-induced immune response of hybrid striped bass (HSB). Nine isonitrogenous and isolipidic diets were prepared including a basal diet and eight treatments consisting of singular or combined additions of $0.5 \%$ AMP and 0.5\% IMP, and combinations of 0.25\% AMP and 0.25\% IMP from each of two suppliers, Sigma-Aldrich and Chem-Impex. The first experiment included two 9-weeks feeding trials in which triplicate groups of HSB $(\sim 9.7 \mathrm{~g})$ were fed the experimental diets. Dietary $0.5 \%$ IMP (C-Impex) showed significantly higher stimulation index of B-lymphocyte proliferation compared with other dietary treatments. However, no significant synergistic effects between AMP and IMP supplementation were observed in production performance. In a subsequent experiment, HSB ( $~ 59.3 \mathrm{~g}$ ) were fed the diets described previously for 2 weeks and subjected to a standardized acute stress challenge. Fish fed some of the dietary nucleotide treatments had significant enhancement of innate immunity at 0.5 and $12 \mathrm{hr}$ poststress challenge compared with those fed the basal diet. Dietary $0.5 \%$ AMP (Chem-Impex) provided the best capability for enhancing innate immunity during poststress and resistance to stress-induced immunosuppression.
\end{abstract}

